Martin Dietrich und Oliver Grapp

\title{
Qualitätsinformationen von Krankenhäusern
}

\author{
Eine Untersuchung ihrer Relevanz und Anforderungen \\ aus Patientensicht
}

Gesundheitsökonomie, Informationspolitik im Gesundheitswesen; Krankenhausmanagement; Patienten-Wahlverhalten; Qualitätsinformationen von Krankenhäusern; Wettbewerb

Unter den Bedingungen eines sich verschärfenden Wettbewerbs unter Krankenhäusern gilt die Qualität als eines der wesentlichsten Wettbewerbsargumente, um Einfluss auf das Wahlverhalten von Patienten zur Sicherung der Auslastung zu nehmen. Neben der Sicherstellung einer möglichst hohen Qualität sehen sich die Krankenhäuser zusätzlich vor die Herausforderung gestellt, die von ihnen geleistete Qualität wirkungsvoll zu kommunizieren. Da insbesondere die Patienten als die eigentlichen Nutzer des Gesundheitssystems letztendlich die relevante Zielgruppe darstellen, ist von grundlegendem Interesse, welche Bedeutung Qualitätsinformationen im Wettbewerb unter Krankenhäusern aufgrund des Wahlverhaltens von Patienten haben, welche Informationen über die Qualität der Leistungen von Patienten nachgefragt werden und welche Informationskanäle von diesen bevorzugt genutzt werden möchten. Weiterhin lässt sich die Frage stellen, ob der Schweregrad einer Erkrankung einen Einfluss auf die von den Patienten nachgefragten Qualitätsinformationen und bevorzugten Informationskanäle hat. Hierzu werden die Ergebnisse einer Befragung unter 240 potenziellen Patienten bei niedergelassenen Ärzten dargestellt.

\section{Problemstellung}

Die Qualität von Krankenhausleistungen in einem Umfeld zunehmenden Wettbewerbs gehört zu den zentralen Erfolgsfaktoren von Krankenhäusern und hat bereits einige Forschungsarbeiten initiiert. ${ }^{1}$ Einerseits liegt die Bedeutung der Qualität für Krankenhäuser im rechtlichen Rahmen der gesetzlichen Krankenversicherung begründet, der Kranken-

1 Vgl. z.B. Olandt/Benkenstein (1999), S. 112; Hribek/Schmalen (2000), S. 208 f. 
häuser zur Qualitätssicherung und deren Nachweis verpflichtet. ${ }^{2}$ Da für Krankenhäuser eine marktorientierte Unternehmensführung insbesondere in der Produkt- bzw. Leistungspolitik zu sehen ist, übt andererseits der durch ordnungspolitische Rahmenbedingungen zunehmend intensivierte Wettbewerb Druck auf die Krankenhäuser aus, auch im Bereich der Qualität vermehrt marktorientiert zu handeln. ${ }^{3}$

Neben der Sicherung und Entwicklung der Qualität ist aber auch von Bedeutung, wie die geleistete Qualität von Krankenhäusern wirkungsvoll kommuniziert und signalisiert werden kann. Dabei geht es um das Problem, welche Informationen Krankenhäuser als Qualitätssignale senden und welcher Kanäle sich die Krankenhäuser zur Übermittlung dieser Signale an die relevanten Zielgruppen bedienen sollen. ${ }^{4}$ Für Krankenhäuser stellt eine hohe Qualität des Leistungsangebots zwar eine notwendige Bedingung ihrer Existenz dar, die alleine aber für ihr Bestehen nicht hinreichend ist, wenn es Krankenhäusern nicht gelingt, eine hohe Qualität auch wirkungsvoll, d.h. überzeugend zu vermitteln. Erschwerend bei dieser Fragestellung gilt für Krankenhäuser, dass ihre Kommunikationspolitik restriktiven gesetzlichen Einschränkungen unterliegt. ${ }^{5}$

Die Frage nach der Gestaltung der Qualitätsinformationen gilt dabei unabhängig von der Verpflichtung der Krankenhäuser, Qualitätsberichte veröffentlichen zu müssen, ${ }^{6}$ die auch als Grundlage zur Patienteninformation dienen sollen. ${ }^{7}$ Aufgrund dieser Qualitätsberichte sollen Krankenkassen und Kassenärztliche Vereinigungen ihre Vertragsärzte und Versicherten über die Qualitätsmerkmale der Krankenhäuser vergleichend informieren können. ${ }^{8}$ Insofern die rechtlichen Vorgaben zur Qualitätsberichterstattung aber für alle Krankenhäuser gleichermaßen gelten, können sich Krankenhäuser damit nicht wirkungsvoll profilieren. ${ }^{9}$ In welcher Weise die Pflicht-Qualitätsinformationen von Patienten als letztendliche Zielgruppe tatsächlich genutzt werden (können) und wie sich die Nutzung dieser Informationen letztendlich auf das Wahlverhalten der Patienten auswirkt, muss nach dem Stand der Dinge für Deutschland bisher noch als weitgehend unbestimmt bezeichnet werden.

Zunächst stellt sich für Krankenhäuser damit die Frage, auf welche Weise Qualitätsinformationen einen wettbewerbsrelevanten Einfluss ausüben können. Die Nutzbarmachung dieser Wirkungsweisen für eine marktorientierte Krankenhausführung macht es notwendig, den Einfluss von Qualitätsinformationen auf das Wahl- und Informationssuchverhalten von Patienten aufzudecken. Somit lässt sich als erste Forschungsfrage der vorliegenden Untersuchung formulieren:

2 Vgl. Artikel 1, Ziffer 54 GKV-Reform 2000. Hier wurden die rechtlichen Rahmenbedingungen und Anforderungen an die Qualitätssicherung bei zugelassenen Krankenhäusern durch die Einführung des § 137 SGB V bestimmt, $\S \S 112,113$ SGB V.

3 Vgl. Tscheulin/Helmig/Moog (2001), S. 451; Lüngen/Lauterbach (2002), S. 165. Vgl. auch z.B. Tscheulin/Helmig (2000); Adam/Gorschlüter (1999); Hribek/Schmalen (2000); Etienne (2000); Schmutte (1998); Olandt (1998); Viethen (1995); Kaltenbach (1991, 1992, 1993).

4 Vgl. Schrappe (1999), S. 511.

5 Vgl. Tscheulin/Helmig (1996), S. $1358 \mathrm{f}$.

$6 \S 137$ Abs. 1 Ziffer 6 SGB V.

7 Vgl. Tuschen/Trefz (2004), S. 121.

$8 \S 137$ SGB V; vgl. auch Tuschen/Trefz (2004), S. 121.

9 Vgl. Oberender/Daumann (1996), S. 568. 
- Wie lässt sich die Relevanz von Qualitätsinformationen für den Wettbewerb im Krankenhauswesen konzeptionell und empirisch hinsichtlich des Verhaltens von Patienten als wichtige Zielgruppe von Krankenhäusern begründen?

Können wettbewerbsrelevante Wirkungsmechanismen identifiziert werden, stellt sich für Krankenhäuser darauf aufbauend die Frage, wie Qualitätsinformationen wirkungsvoll als Element ihrer Kommunikation innerhalb der ihnen gegebenen Möglichkeiten eingesetzt werden können. Die anforderungsgerechte Kommunikation der Qualität stellt für Krankenhäuser somit ein spezielles Management-Problem dar, welches dahingehend zu lösen ist, dass sie ihre Qualität möglichst wirkungsvoll auf den von den Patienten bevorzugten Informationskanälen kommunizieren. Dabei kann von Bedeutung sein, dass unterschiedliche Merkmale von Patienten wie z.B. der Schweregrad der Erkrankung einen Einfluss auf Informationsbedürfnisse über die Qualität von Krankenhausleistungen haben können. Somit lässt sich als zweite forschungsleitende Frage formulieren:

- Welche Bedürfnisse hinsichtlich der Art der Informationen und hinsichtlich der Kanäle von Qualitätsinformationen haben Patienten? Unterscheiden sich die Qualitätsbedürfnisse von Patienten in Abhängigkeit von spezifischen Patientenmerkmalen?

\section{Konzeptionelle Grundlagen}

1. Wettbewerbsrelevanz von patientenorientierten Qualitätsinformationen im Krankenhauswesen

Grundlegende Voraussetzung dafür, dass Patienten als wettbewerbsrelevante Zielgruppe für Qualitätsinformationen von Krankenhäusern anzusehen sind, ist, dass der Entscheidungsanteil von Patienten bei der Wahl eines Krankenhauses hinreichend hoch ist. Dabei sind Patienten zwar formal an die Empfehlung eines einweisenden Arztes bezüglich des Krankenhauses weitgehend gebunden. ${ }^{10} \mathrm{Da}$ aber die Patienten die letztentscheidende Instanz für einen Krankenhausaufenthalt und die darin vorgenommenen Eingriffe sind, kann vermutet werden, dass die Wahl eines Krankenhauses zumindest im Falle eines elektiven, d.h. planbaren Aufenthalts in Absprache des einweisenden Arztes mit dem Patienten getroffen wird. ${ }^{11}$ Auch wird durch die zunehmende Entscheidungskompetenz der Patienten innerhalb des Gesundheitswesens ihre Rolle als autonome und wohlinformierte Nutzer des Systems zukünftig weiter verstärkt, indem sie vermehrt auf eigene Kompetenzen beruhende Entscheidungen z.B. in Fragen der Wahl bestimmter Leistungsanbieter

10 Vgl. Neubauer (2004), S. 104; § 39 Abs. 2 SGB V.

11 Insbesondere die Förderung von Einrichtungen zur Patienteninformation durch $\S 65 \mathrm{~b}$ SGB V und die Institutionalisierung einer Patientenvertretung durch die $\S \S 140 \mathrm{f}-\mathrm{h}$ SGB V spiegeln die institutionelle Perzeption der Patienten auf ordnungsrechtlicher Ebene wider. 
treffen werden. ${ }^{12}$ Patienten werden somit noch weiter an Bedeutung als Zielgruppe für wettbewerbsorientierte Aktivitäten der Leistungserbringer in einem stärker am Wettbewerb ausgerichteten Gesundheitssystem gewinnen. Die längerfristige Gestaltung des Leistungsgeschehens von Krankenhäusern als strategisches Element der Krankenhausmanagements wird dabei zunehmend von Wahlentscheidungen der Patienten abhängig sein: „Es ist zu erwarten, dass hierdurch [...] eine Schwerpunktbildung bei Krankenhäusern (Spezialisierung) infolge der Krankenhauswahl der Patienten begünstigt wird." “13 Die Frage nach der Entscheidungsinstanz bei der Wahl von Krankenhäusern ist bereits Gegenstand verschiedener Untersuchungen. Dabei ist der Entscheidungsanteil der Patienten im Vergleich zum Entscheidungsanteil der Ärzte von besonderem Interesse. Haubrock, Meiners und Albers stellen für Deutschland fest, dass potenzielle Patienten in 33\% der Fälle entscheiden, welches Krankenhaus gewählt wird. ${ }^{14}$ Ein Haus- oder Facharzt hat in $54 \%$ der Fälle ein Krankenhaus empfohlen oder gar die Wahl des Krankenhauses getroffen. Nach Meurer wählen $24 \%$ der Patienten selbst das Krankenhaus und $21 \%$ der Patienten mit dem Arzt gemeinsam. ${ }^{15}$ Im US-amerikanischen Raum ermitteln z.B. Kurz und Wolinsky, dass $41 \%$ der befragten Patienten selbst oder ein Mitglied ihres Haushalts die Wahl des Krankenhauses getroffen haben, Wallace ermittelt $35 \%$ unter den befragten Patienten als Entscheidungsträger der Krankenhauswahl. Noch höhere Anteile der Patienten an der Entscheidung bei der Krankenhauswahl berichten Sloane, Tidwell und Horsfield, die ermitteln, dass $65 \%$ der befragten Ärzte den Patienten die letztendliche Entscheidungskompetenz für die Wahl eines Krankenhauses einräumen. ${ }^{16}$ Der Entscheidungsanteil von Patienten bei der Krankenhauswahl stellt somit eine relevante und wesentliche Größe dar, weshalb die Entscheidungsunterstützung der Patienten durch Qualitätsinformationen durch Krankenhäuser als wettbewerbsrelevant aufzufassen ist.

Die Wettbewerbsrelevanz von Informationen über die Qualität von Krankenhäusern kann damit begründet werden, dass die Nutzung von Krankenhausinformation durch potenzielle Patienten und eine daraus resultierende bewusste Auswahl eines Krankenhauses zu einer höheren Kompatibilität zwischen dem Anforderungsprofil des Falles eines Patienten und dem Leistungsprofil des ausgewählten Krankenhauses führt. Aufgrund dieser höheren Kompatibilität bei der Integration des Patienten als Fall in die Behandlungsstrukturen und spezifischen Kompetenzen des Krankenhauses kann angenommen werden, dass dadurch bessere Ergebnisse erzielt werden können. Zusätzlich kann davon ausgegangen werden, dass die über die Leistungsfähigkeit von Krankenhäusern informierten (potenziellen) Patienten solche Krankenhäuser wählen, die allgemein eine hohe Behandlungsqualität aufweisen. ${ }^{17}$

12 Zur Nutzerkompetenz als Problem eines zielorientiert konzeptionierten Gesundheitssystems vgl. z.B. den Sachverständigenrat der Konzertierten Aktion im Gesundheitswesen (SKAiG) (2001), S. 144 ff.; Hibbard/Slovic/Jewett (1997), S. 395. Zum Nutzen höherer Eigenverantwortung in der medizinischen Versorgung vgl. z.B. May/Wasem (2003); bezüglich des Wunsches von Patienten nach Autonomie und Einbeziehung in Entscheidungen vgl. z.B. Johnson/Ramaprasad (2000); Nease/Brooks (1995); Ende u.a. (1989).

13 Tuschen/Trefz (2004), S. 121.

14 Vgl. Haubrock/Meiners/Albers (1998), S. 105.

15 Vgl. Meurer (1998), S. 312.

16 Vgl. Sloane/Tidwell/Horsfield (1999), S. 60.

17 Vgl. z.B. Mukamel/Mushlin (1998). 
Eine weitere Ursache für die subjektive Bewertung von Behandlungsergebnissen durch Patienten lässt sich auch verhaltenstheoretisch damit begründen, dass das Informationsverhalten bzw. der Informationsstand von Patienten einen positiven Einfluss auf das subjektiv wahrgenommene Behandlungsergebnis hat. ${ }^{18}$ Diesem Zusammenhang kann die Selbstwahrnehmungstheorie als Erklärungsmöglichkeit zugrunde gelegt werden, die besagt, dass informiert getroffene Entscheidungen aufgrund der höheren Einbezogenheit positive Attributionen und damit eine höhere Zufriedenheit als subjektives Urteil der Patienten als Entscheider über die Leistungsfähigkeit eines Krankenhauses auslösen können. ${ }^{19}$ Aus diesen Überlegungen ergibt sich die Hypothese:

\section{H1: Ein höherer Informationsstand von Patienten bezüglich der Leistungen von Kran- kenhäusern hat einen positiven Einfluss auf das durch die Patienten subjektiv be- wertete Ergebnis von Behandlungen im Krankenhaus.}

Weiter kann davon ausgegangen werden, dass sich als Folge einer hohen Zufriedenheit mit den Leistungen eines Krankenhauses die Bindung von Patienten an das Krankenhaus erhöht und sich gleichzeitig die Wechselabsichten verringern, ${ }^{20}$ was unmittelbar relevant für das zukünftige Informationssuchverhalten von Patienten ist. Die Bindungswirkung der Kunden- bzw. Patientenzufriedenheit wurde in der Literatur bereits intensiv erforscht und kann empirisch als weitgehend abgesichert angesehen werden. ${ }^{21}$ Umgekehrt bedeutet dies, dass bei einer als nicht zufrieden stellend wahrgenommenen Leistung Wettbewerbsdruck auf Krankenhäuser ausgeübt wird, da die Wechselabsichten von Patienten zunehmen $^{22}$ und dadurch die Auslastung entsprechender Krankenhäuser gefährdet werden kann.

Sind Patienten aufgrund von schlechten Erfahrungen in einem bestimmten Krankenhaus wechselwillig, kann angenommen werden, dass bei einem erneuten Krankenhausaufenthalt im Vorfeld der Behandlung intensiver nach Informationen über die Qualität anderer Krankenhäuser gesucht wird. Anders herum kann davon ausgegangen werden, dass Patienten, die gute Erfahrungen gemacht haben und die demzufolge keine erhöhte Wechselbereitschaft aufweisen, auch weniger intensiv nach Informationen suchen. Von dem Informationssuchverhalten wechselwilliger Patienten können andere Krankenhäuser insofern profitieren, als sie bei einer entsprechend wirkungsvollen Kommunikation ihrer Qualität diese wechselwilligen und Informationen suchenden Patienten an sich ziehen können.

Darüber hinaus kann angenommen werden, dass eine als nicht zufrieden stellend wahrgenommene Leistung auch ein direktes Informationssuchverhalten auslöst, welches sich in Anlehnung an die Attributionstheorie erklären lässt. Die Attributionstheorie hat sub-

18 Vgl. z.B. Helmig/Dietrich (2001); Woodside/Sertich/Chakalas (1987), S. $61 \mathrm{f}$.

19 Vgl. z.B. Bem (1972).

20 Vgl. Helmig/Dietrich (2001); Woodside/Frey/Daly (1989).

21 Vgl. Kamakura u.a. (2002).

22 Vgl. z.B. Zeithaml/Berry/Parasuraman (1996), S. 32 und 33; Boulding u.a. (1993), S. 13.; Morwitz/Schmittlein (1992). 
jektive Ursache-Wirkungs-Zusammenhänge zum Gegenstand und beschreibt vergleichsweise rationale Prozesse der Informationsverarbeitung im Alltagsdenken. ${ }^{23}$ Kennzeichnend für die subjektive Verknüpfung von möglichen Ursachen mit subjektiv vermuteten Wirkungen im attributionstheoretischen Sinne ist, dass subjektiv zugrunde gelegte Kausalzusammenhänge erst im Nachhinein gesucht werden. Daher kann vermutet werden, dass zur Begründung eines nicht zufrieden stellenden Krankenhausaufenthaltes Informationen über die Qualität von Krankenhäusern gesucht werden, auch ohne dass ein konkreter Bedarf und eine aktuelle Wechselabsicht vorliegen müssen. Daraus lässt sich folgende Hypothese ableiten:

H2: Die subjektive Ergebniswahrnehmung hat einen direkten Effekt auf die Wechselabsicht sowie einen direkten als auch einen indirekten Effekt auf das zukünftige Informationssuchverhalten von potenziellen Krankenhauspatienten.

Nachfolgende Abbildung 1 stellt die in $H 1$ und $H 2$ unterstellten Zusammenhänge im Überblick dar.

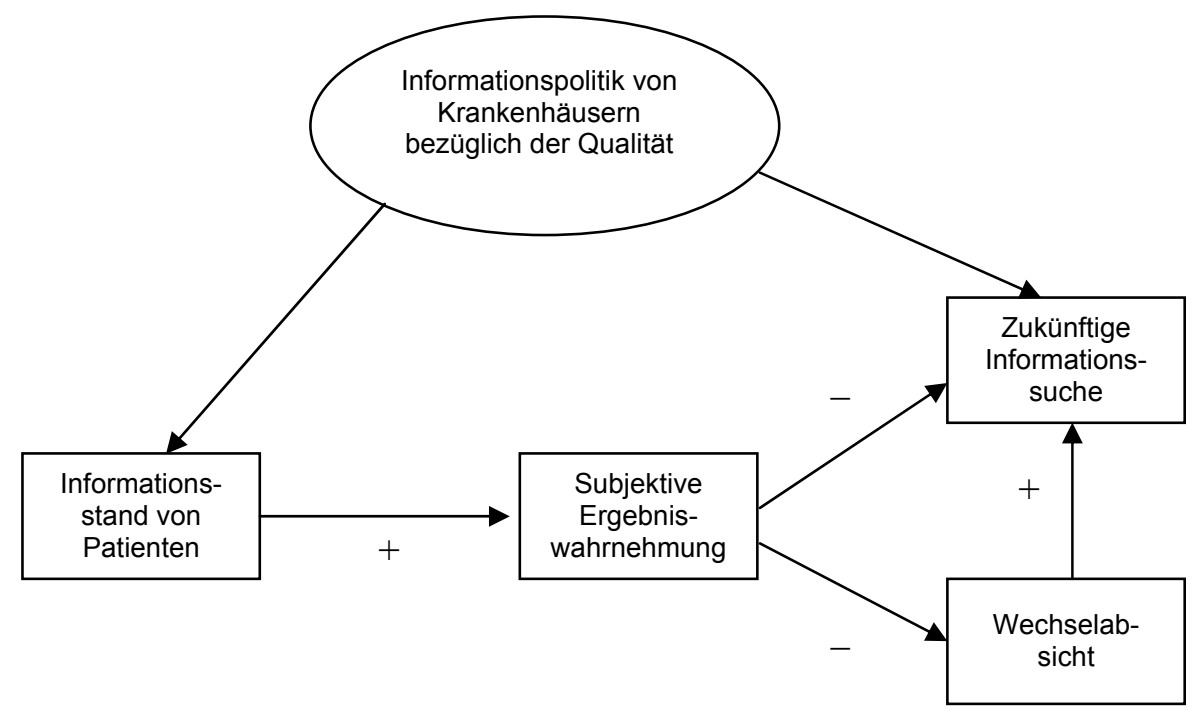

Abb. 1: Unterstellte Wirkungszusammenhänge zwischen Informationsstand, subjektivem Outcome und zukünftigem Informationssuchverhalten von Patienten

Quelle: Eigene Darstellung 
2. Qualitätsinformationen von Krankenhäusern und Informationsbedürfnisse von potenziellen Krankenhauspatienten

Innerhalb der entscheidungsrelevanten Informationen wird insbesondere den Informationen über die Qualität der Leistung ${ }^{24}$ eine zentrale Rolle zugesprochen. ${ }^{25}$ Der Entwicklung von kommunizierbaren Indikatoren der Leistungsfähigkeit und Qualität von Krankenhäusern und deren Veröffentlichung wurde insbesondere im angloamerikanischen Raum eine hohe Aufmerksamkeit gewidmet. ${ }^{26}$ Inzwischen stellt sich jedoch die Frage, ob die Informationen in Form von „Quality Report Cards", „Health Care Report Cards" oder „League Tables“ ihrer Art nach die Bewertungs- und Entscheidungsprozesse von Patienten tatsächlich unterstützen können. ${ }^{27}$ Die Ursache möglicher Probleme der Wirksamkeit dieser Qualitätsinformationen kann darin gesehen werden, dass die Indikatoren meist aus der Perspektive der professionellen Fachvertreter oder der Leistungsfinanzierer bzw. Kostenträger entwickelt wurden und mögliche Anforderungen aus Patientensicht nicht explizit berücksichtigt werden. ${ }^{28}$ Wie Untersuchungen über die Nutzung von entsprechender Indikatoren durch (potenzielle) Patienten ergeben, scheint das Verständnis der vornehmlich fachspezifisch bzw. klinisch konzipierten Indikatoren unter Patienten sehr gering zu sein. ${ }^{29}$ Daraus resultiert zwangsläufig, dass diese Informationen von Patienten nicht zur Unterstützung bei der Wahl von Leistungsanbietern bzw. Krankenhäusern genutzt werden können und diese somit eigentlich keine Informationen sind. Zudem sind Qualitätsindikatoren z.B. über Komplikationen oder über die Mortalität eines spezifischen Krankenhauses nicht ohne weiteres vergleichend interpretierbar. ${ }^{30}$ Eine ungeeignete Konzipierung von Qualitätsinformationen von Krankenhäusern verliert damit ihre Funktion, die Entscheidungskompetenz der Patienten und damit ein stärker an den Nachfragern ausgerichteten Wettbewerb unterstützen zu können.

Daraus wird ersichtlich, dass Qualitätsinformationen von Krankenhäusern nur genutzt werden können, wenn sie mit ihrer Informationspolitik den Informationsbedürfnissen der Patienten entsprechen. Daher müssen die Informationsbedürfnisse und -anforderungen der Patienten ein zentraler Bestandteil der Überlegungen zur Qualitäts-Informationspolitik von Krankenhäusern sein. An dieser Stelle soll als Informationsbedürfnis die Art von Qualitätsinformationen und die bevorzugten Kanäle und Quellen verstanden werden, über die bzw. von denen Qualitätsinformationen empfangen werden. Dabei ist auch die Frage wichtig, welchen Einfluss patientenspezifische Merkmale auf das Informationsbedürfnis haben, wobei hier der Schweregrad von Erkrankungen als ein relevantes Kriterium angesehen werden soll.

24 Vgl. z.B. Javalgi/Rao/Thoms (1991).

25 Vgl. z.B. Lane/Lindquist (1988); Luft u.a. (1990); Robinson/Brodie (1997); Taylor/Capella (1996); Saeed (1998).

26 Vgl. z.B. Nutley/Smith (1998); Lubalin/Harris-Kojetin (1999).

27 Vgl. z.B. Smith (1995); Hibbard/Sofaer/Jewett (1996); Hibbard/Jewett (1997); McKee (1997); McKee/Hunter (1995); Mukamel/Mushlin (2001).

28 Vgl. ähnlich z.B. Hibbard/Sofaer/Jewett (1996), S. 95; Galvin (1998), S. 30.

29 Vgl. z.B. Chassin/Hannan/DeBuono (1996), S. 96; Cheng/Ho/Chung (2002).

30 Vgl. z.B. Davis/Marshall (1999), S. 1639 f.; Halm/Chassin (2001), S. 692 f. 
Um den Bedürfnissen der Patienten hinsichtlich der Qualitätsinformationen über Krankenhäuser zu entsprechen, liegt es zunächst nahe, die Qualitätsinformationen über die Leistungsfähigkeit eines Krankenhauses gemäß dem Ansatz von Donabedian zu strukturieren. ${ }^{31}$ Die Validität der Konzeptualisierung der Qualität von Krankenhausleistungen nach den Dimensionen der Potenzial-, Prozess- und Ergebnisqualität gilt als empirisch gut abgesichert. ${ }^{32}$ Dementsprechend erscheint es sinnvoll, auch Informationen über die Leistungsqualität eines Krankenhauses anhand der Potenzial-, Prozess- und Ergebnisdimension zu strukturieren. Daraus lässt sich die Hypothese ableiten:

\section{H3: Die Wahrnehmung der Qualitätsinformationen über Leistungen von Krankenhäu- sern entsprechen der Strukturierung der Qualität von Krankenhausleistungen an- hand der Potenzial-, Prozess- und Ergebnisdimension.}

Zudem kann davon ausgegangen werden, dass die Informationsbedürfnisse von Patienten je nach Schweregrad der Erkrankung unterschiedlich sind. So mögen für Patienten mit einer relativ leichten Erkrankung die nicht medizinischen Merkmale eines Krankenhauses, wie z.B. die Lage und Verkehrsanbindung des Krankenhauses oder sonstige Ausstattungsmerkmale, wichtiger sein als für Patienten mit einer schwereren Erkrankung, für die die medizinischen und pflegerischen Aspekte der Behandlung im Vordergrund stehen dürften. Es kann daher erwartet werden, dass entsprechende Informationsinhalte über die verschiedenen Qualitätsaspekte unterschiedlich in ihrer Bedeutung gewichtet werden.

Dies sollte sich auch in der Bedeutung der entsprechenden Informationsquellen und kanäle widerspiegeln, auf denen Qualitätsinformationen übermittelt werden. Es erscheint plausibel, dass Patienten mit schwereren Erkrankungen Informationsquellen und -kanäle mit direktem medizinischem und pflegerischem Bezug, wie z.B. Krankenhäuser, Hausund Fachärzte, unabhängige medizinische Beratungsinstitute oder Krankenkassen, höher gewichten als andere Informationsquellen wie z.B. Freunde, Bekannte und Verwandte, die Presse oder andere Medien. Anders kann es sich für leichtere Fälle darstellen, bei denen die Gewichtung anderer als medizinisch geprägter Informationsquellen höher ausfallen mag. Aus diesen Überlegungen lässt sich die Hypothese formulieren:

H4: Je höher der Schweregrad der Erkrankung von Patienten ist, desto wichtiger sind sowohl medizinisch-pflegerische Informationsbestandteile über die Qualität als auch medizinisch-pflegerisch orientierte Quellen bzw. Kanäle von Qualitätsinformationen.

31 Vgl. Donabedian (1980); in einer empirischen Analyse z.B. Helmig/Dietrich (2001), S. 320 f.

32 Vgl. z.B. Helmig/Dietrich (2001). 


\section{Empirische Untersuchung}

\section{Untersuchungsdesign}

Die empirische Untersuchung zur Überprüfung der oben formulierten Hypothesen basiert auf einer schriftlichen Befragung, die unter 240 potenziellen Krankenhauspatienten im süddeutschen Raum im Herbst 2003 durchgeführt wurde. Die Befragung erfolgte in Arztpraxen, bei der den Patienten während der Wartezeit Fragebögen direkt ausgegeben wurden. Die Auswahl der Befragten wurde anhand eines Quotenverfahrens durchgeführt, welches die soziodemographischen Merkmale der in Deutschland lebenden Bevölkerung berücksichtigte. Vor dem Einsatz des Erhebungsinstruments wurde eine erste Version des Fragebogens einem Pretest unterzogen, der zu geringen Änderungen in der Formulierung einzelner Fragen führte. Zusätzlich wurde der Fragebogen medizinischen Experten vorgelegt, deren Hinweise zu den Beispielen in den unten beschriebenen Szenarien aufgenommen wurden.

Um der Frage nach der Wettbewerbsrelevanz von Qualitätsinformationen für potenzielle Patienten nachzugehen, wurden geschlossene Fragen zum Informationsstand bezüglich der Leistungen von Krankenhäusern, der Zufriedenheit mit dem letzten Krankenhausaufenthalt, des zukünftigen Informationssuchverhaltens sowie zu den Wechselabsichten gestellt. Als Bewertungsgrundlage sollte hierbei der letzte Krankenhausaufenthalt herangezogen werden, den die Befragten absolviert hatten. Um die Wechselabsicht zu untersuchen, wurde gefragt, ob bei einer gleichen Erkrankung das Krankenhaus des letzten Aufenthalts wieder gewählt werden würde. Alle Antworten wurden anhand von fünfer Ratingskala erfasst.

Die verwendeten Fragen über die Entscheidungsgründe der Krankenhauswahl sowie zu den Qualitätsinformationen von Krankenhäusern orientierten sich inhaltlich an der bestehenden Literatur. ${ }^{33}$ Dabei wurden die Fragen zu den Qualitätsinformationen entsprechend der Systematisierung der drei Qualitätsdimensionen Potenzial-, Prozess- und Ergebnisqualität strukturiert (vgl. Tabelle 1). ${ }^{34} \mathrm{Zu}$ diesen Aspekten wurde gefragt, für wie wichtig Informationen über diese Leistungsmerkmale für die Wahl eines bestimmten Krankenhauses angesehen werden, wobei auch diese Antworten anhand von fünfer Ratingskalen erfasst wurden.

33 Vgl. z.B. Malhotra (1983); Nix/Gibson (1987); Javalgi u.a. (1991); Beumers (1997); Borges (2003).

34 Vgl. Donabedian (1966), S. 166 ff.; Donabedian (1980), S. 79 ff. 


\begin{tabular}{|c|c|c|}
\hline \multicolumn{3}{|c|}{ Qualitätssignale } \\
\hline Strukturqualität & Prozessqualität & Ergebnisqualität \\
\hline $\begin{array}{ll}\text { - } & \text { Lage der Klinik } \\
\text { - } & \text { Qualifikation der Ärzte } \\
\text { - } & \text { Nicht-medizinische Ausstat- } \\
\text { tung (z.B. Zimmerausstattung) } \\
\text { - } & \text { Mitarbeiterzufriedenheit } \\
\text { - } & \text { Verkehrsanbindung der Klinik } \\
\text { - } & \text { Allgemeine Krankenhaus- } \\
& \text { beschreibung } \\
\text { - } & \text { Sonstige Ausstattungen des } \\
& \text { Krankenhauses (z.B. Kino) }\end{array}$ & 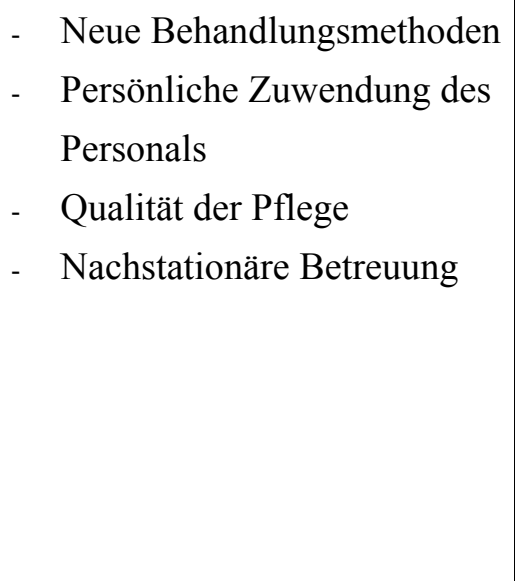 & $\begin{array}{ll}\text { - } & \text { Zufriedenheit ehemaliger } \\
& \text { Patienten } \\
\text { - } & \text { Mortalitätsrate } \\
\text { - } & \text { Stationäre Wiedereinweisung } \\
\text { - } & \text { Krankenhaus-Rankings } \\
- & \text { Krankenhausinterne Infekti- } \\
& \text { onsrate }\end{array}$ \\
\hline
\end{tabular}

Tab. 1: Systematisierung der untersuchten Qualitätssignale

Quelle: $\quad$ Eigene Darstellung

Um den Einfluss der Schwere der Erkrankung untersuchen zu können, wurden die Befragten in zwei gleich große Gruppen geteilt und ihnen jeweils zwei unterschiedliche Szenarien als Grundlage für ihre weiteren Antworten geschildert. Als das Szenario des „leichten Falls" wurde allgemein von einer leichten Operation gesprochen, die ein sehr geringes Risiko aufweist und nach der der ursprüngliche Gesundheitszustand als wiederhergestellt bezeichnet werden kann. Als Beispiel wurde eine Gallenstein-Operation angeführt. Das Szenario eines „schweren Falls“ wurde so dargestellt, dass die damit verbundene Operation ein hohes Risiko aufweist und der ursprüngliche Gesundheitszustand wahrscheinlich nicht wiederhergestellt werden kann, wofür das Beispiel einer Herzoperation genannt wurde.

Hinsichtlich der bevorzugten Informationskanäle wurden die Probanden gefragt, ob sie sich bei der Notwendigkeit einer stationären Behandlung aktiv über Krankenhäuser informieren würden, und wenn ja, über welche Kanäle bzw. Quellen. Als Alternativen wurden hierbei die Antworten „Presse“, „Gespräch beim Arzt“, „Freunde, Bekannte und Verwandte“, „Recherche im Internet“, „Anfordern von Infopost von Krankenhäusern“, „Besuch eines Tages der offenen Tür von Krankenhäusern“ sowie „sonstiges“ vorgegeben. Zusätzlich wurde anhand einer offenen Frage erhoben, wie bzw. von wem die Probanden gerne zusätzlich Informationen erhalten würden, ohne diese explizit anzufordern.

\section{Ergebnisse}

\section{a) Deskriptive Analyse}

Die Zusammensetzung der Stichprobe $(\mathrm{n}=240)$ ist Tabelle $2 \mathrm{zu}$ entnehmen, wobei jeweils die Anteile für die Fälle des „leichten“ Szenarios $(\mathrm{n}=120)$, des „schweren“ Sze- 
narios $(\mathrm{n}=120)$ und für beide Fälle gemeinsam angegeben wurden. Es kann hieraus ersehen werden, dass die Repräsentativität gemäß der Merkmalsverteilung der Bevölkerung Deutschlands weitgehend hergestellt werden konnte. Insgesamt gaben 163 bzw. 68 \% der Befragten an, dass sie bereits einmal ein Krankenhaus für einen planbaren Eingriff aufgesucht hatten.

\begin{tabular}{|c|c|c|c|c|c|c|}
\hline Alter & $\begin{array}{c}15-24 \\
13,3 \%-9,2 \% \\
11,3 \%\end{array}$ & \multicolumn{2}{|c|}{$\begin{array}{c}25-44 \\
40,8 \%-49,2 \% \\
45,0 \%\end{array}$} & \multicolumn{2}{|c|}{$\begin{array}{c}45-64 \\
31,7 \%-29,2 \% \\
30,4 \% \\
\end{array}$} & $\begin{array}{c}65 \text { und darüber } \\
14,2 \%-12,5 \% \\
13,3 \%\end{array}$ \\
\hline Geschlecht & \multicolumn{3}{|c|}{$\begin{array}{c}\text { männlich } \\
9,2 \%-48,3 \% \\
48,8 \% \\
\end{array}$} & \multicolumn{3}{|c|}{$\begin{array}{c}\text { weiblich } \\
50,8 \%-51,7 \% \\
51,2 \% \\
\end{array}$} \\
\hline $\begin{array}{l}\text { Staatsan- } \\
\text { gehörigkeit }\end{array}$ & \multicolumn{3}{|c|}{$\begin{array}{c}\text { deutsch } \\
94,2 \%-93,3 \% \\
93,8 \%\end{array}$} & \multicolumn{3}{|c|}{$\begin{array}{c}\text { andere } \\
5,8 \%-6,7 \% \\
6,3 \%\end{array}$} \\
\hline \multirow[b]{2}{*}{$\begin{array}{l}\text { Beruflicher } \\
\text { Status }\end{array}$} & $\begin{array}{c}\text { Selbständige } \\
9,2 \%-9,2 \% \\
9,2 \%\end{array}$ & \multicolumn{2}{|c|}{$\begin{array}{c}\text { Beamte } \\
3,3 \%-4,2 \% \\
3,8 \% \\
\end{array}$} & \multicolumn{2}{|c|}{$\begin{array}{c}\text { Angestellte } \\
35,8 \%-33,3 \% \\
34,6 \% \\
\end{array}$} & $\begin{array}{c}\text { Arbeiter } \\
10,8 \%-13,3 \% \\
12,1 \% \\
\end{array}$ \\
\hline & $\begin{array}{c}\text { Auszubildende } \\
\begin{array}{c}3,3 \%-2,5 \% \\
2,9 \%\end{array}\end{array}$ & $\begin{array}{c}\text { Studenten } \\
4,2 \%-5,0 \% \\
4,6 \%\end{array}$ & \multicolumn{2}{|c|}{$\begin{array}{l}\text { Arbeitslose } \\
6,7 \%-4,2 \% \\
5,4 \%\end{array}$} & $\begin{array}{c}\text { Rentner } \\
14,2 \%-15,8 \\
\% \\
15,0 \% \\
\end{array}$ & $\begin{array}{c}\text { sonstige } \\
12,5 \%-12,5 \\
\% \\
12,5 \%\end{array}$ \\
\hline $\begin{array}{l}\text { Nettoein- } \\
\text { kommen* }\end{array}$ & \multicolumn{2}{|c|}{$\begin{array}{c}<1500 \text { Euro } \\
40,8 \%-40,8 \% \\
40,8 \% \\
\end{array}$} & \multicolumn{2}{|c|}{$\begin{array}{c}1500-2500 \text { Euro } \\
39,2 \%-29,2 \% \\
34,2 \%\end{array}$} & \multicolumn{2}{|c|}{$\begin{array}{c}>2500 \text { Euro } \\
11,7 \%-15,0 \% \\
13,3 \%\end{array}$} \\
\hline Bildung & $\begin{array}{c}\text { Volks-/Hauptsch } \\
\text { abschluss } \\
37,5 \%-37,5 \% \\
37,5 \%\end{array}$ & $\begin{array}{r}\text { Realschul } \\
\text { Absch } \\
19,2 \%- \\
20,0\end{array}$ & $\begin{array}{l}g l w . \\
8 \%\end{array}$ & $\begin{array}{r}\text { (Fach } \\
41,7\end{array}$ & $\begin{array}{l}\text { Hochschul- } \\
\text { reife } \\
0-39,2 \% \\
0,4 \%\end{array}$ & $\begin{array}{c}\text { sonstige } \\
\begin{array}{c}1,7 \%-2,5 \% \\
2,1 \%\end{array}\end{array}$ \\
\hline $\begin{array}{c}\text { Kranken- } \\
\text { versicherung }\end{array}$ & \multicolumn{3}{|c|}{$\begin{array}{c}\text { gesetzlich versichert } \\
84,2 \%-80,8 \% \\
82,5 \%\end{array}$} & \multicolumn{3}{|c|}{$\begin{array}{c}\text { nicht gesetzlich versichert } \\
14,2 \%-18,3 \% \\
16,3 \%\end{array}$} \\
\hline
\end{tabular}

* Summe $88,3 \%<100 \%$ aufgrund von Non-Response

Tab. 2: Zusammensetzung der Befragten nach soziodemographischen Merkmalen, $n=240$

Quelle: Eigene Darstellung

Für die Analyse des allgemeinen Informationsstands der Befragten über medizinische und nicht-medizinische Leistungen von Krankenhäusern wurden lediglich die Aussagen der 163 Befragten berücksichtigt, die bereits einen stationären Krankenhausaufenthalt absolviert haben, der kein Notfall war. Die deskriptive Analyse ergab, dass der allge- 
meine Informationsstand der Befragten über Krankenhäuser nicht sehr hoch ausgeprägt ist. Mit 33,8 \% gaben deutlich mehr Befragte an, sehr schlecht oder schlecht informiert zu sein, verglichen mit $22,7 \%$ der Befragten, welche angaben, gut oder sehr gut informiert zu sein. Der mit Abstand größte Anteil von 43,6 \% der Befragten gab an, weder gut noch schlecht informiert zu sein. Von dem größeren Teil der Befragten $(50,3 \%)$ wurde angegeben, dass sie sich bei einem erneuten Krankenhaus intensiver um Informationen bemühen würden, während lediglich $22 \%$ angaben, sich eher weniger informieren zu wollen. 71,1 \% der Befragten sahen es als unwahrscheinlich an, dass sie bei einem erneuten Bedarf ein anderes Krankenhaus aufsuchen würden, während dies für 14,1\% eher wahrscheinlich ist. Gemäß der Antworten auf die Frage, wer die Wahl des Krankenhauses bestimmt, beabsichtigt mit 44,6\% der überwiegende Teil der Befragten, selbst die Wahl des Krankenhauses im Falle eines Eingriffes zu wählen. Mit deutlichem Abstand folgen Hausärzte $(27,5 \%)$ und Fachärzte $(25,0 \%)$. Damit wird deutlich, dass Patienten als Zielgruppe für Qualitätsinformationen von Krankenhäusern eine hochrelevante Zielgruppe darstellen. Zusätzlich wurde ermittelt, dass sich insgesamt 80,8\% der Befragten im Falle einer stationären Behandlung aktiv über Krankenhäuser informieren würden. Die Notwendigkeit, für Patienten bedarfsgerechte Qualitätsinformationen bereitzustellen, wird durch diese Ergebnisse unterstrichen.

b) Ergebnisse zur Wettbewerbsrelevanz von patientenorientierten Qualitätsinformationen

Um die in den Hypothesen $H 1$ und $H 2$ formulierten Vermutungen über die Wirkungszusammenhänge zwischen dem Informationsstand von Patienten, der Patientenzufriedenheit, dem zukünftigen Informationsverhalten und den Wechselabsichten zu untersuchen, wurden die Zusammenhänge zwischen diesen Variablen anhand einfacher Korrelationsanalysen gemessen. ${ }^{35}$ Wie Tabelle $3 \mathrm{zu}$ entnehmen ist, kann Hypothese $H 1$, nach der ein hoher Informationsstand eine höhere Zufriedenheit zur Folge hat, als unterstützt angesehen werden. Der positive Zusammenhang ist signifikant, aber nicht sonderlich stark ausgeprägt. Dies kann darauf zurückgeführt werden, dass die Zufriedenheit von Patienten mit einem Krankenhausaufenthalt von einer Vielzahl von Einflussfaktoren abhängig ist, ${ }^{36}$ unter denen die Informiertheit von Patienten vor einem Krankenhausaufenthalt einer von vielen, und wie sich zeigt auch ein signifikanter ist. Ebenso kann die Hypothese $\mathrm{H} 2$ unterstützt werden, gemäß der eine hohe Zufriedenheit negativ auf die Wechselabsichten wirkt und die Wechselabsicht von Patienten einen positiven Einfluss auf das zukünftige Informationssuchverhalten hat. Die gefundenen Zusammenhänge sind signifikant und gemessen an den Korrelationskoeffizienten auch substanziell. Schwächer, aber noch als signifikant zu bezeichnen ist der direkte negative Einfluss der Zufriedenheit auf das In-

35 Die in dieser Untersuchung verwendete Skalierung der Fragen kann als metrisch interpretiert werden, vgl. z.B. Hüttner (1997), S. 109 ff. Aufgrund der Annahme von Wirkungsrichtungen wurde mit dem einseitigen Signifikanzniveau gearbeitet, vgl. z.B. Janssen/Laatz (2003), S. 364.

Vgl. z.B. Helmig/Dietrich (2001). 
formationssuchverhalten, so dass die unterstellten Wirkungszusammenhänge und deren Wirkungsrichtung insgesamt empirisch nachgewiesen werden können. Die konzeptionelle Bedeutung der Informationspolitik aufgrund ihrer hier nachgewiesenen wettbewerbsrelevanten Wirkungsweise wird damit empirisch fundiert.

\begin{tabular}{|c|c|c|}
\hline Unabhängige Variable $\rightarrow$ Abhängige Variable & $\begin{array}{c}\text { Korrelation nach } \\
\text { Brevais-Pearson }\end{array}$ & $\begin{array}{c}\text { Signifikanz } \\
\text { (einseitig) }\end{array}$ \\
\hline Informationsstand $\rightarrow$ Patientenzufriedenheit & 0,181 & 0,010 \\
\hline Patientenzufriedenheit $\rightarrow$ Wechselabsicht & $-0,577$ & 0,000 \\
\hline Patientenzufriedenheit $\rightarrow$ zukünftige Informationssuche & $-0,112$ & 0,077 \\
\hline Wechselabsicht $\rightarrow$ zukünftige Informationssuche & 0,348 & 0,000 \\
\hline
\end{tabular}

Tab. 3: Stärke, Richtung und Signifikanz der Zusammenhänge zwischen dem Informationsstand, der Zufriedenheit, des Informationssuchverhaltens sowie der Wechselabsicht von Krankenhauspatienten

Quelle: Eigene Darstellung

c) Ergebnisse zu den Informationsbedürfnissen von potenziellen Krankenhauspatienten

aa) Wahrgenommene Informationsstruktur über die Qualität von Krankenhausleistungen

Zur Überprüfung der in $H 3$ formulierten Vermutung, dass die Wahrnehmung der Qualitätsinformationen über Leistungen von Krankenhäusern der Strukturierung der Qualität von Krankenhausleistungen gemäß der Potenzial-, Prozess- und Ergebnisdimension entsprechen, wurde eine explorative Faktorenanalyse anhand der Antworten zur Wichtigkeit der abgefragten Qualitätsinformationen durchgeführt (Kaiser-Meyer-Olkin-Kriterium 0,825). ${ }^{37}$ Insgesamt wurden 4 Faktoren mit Eigenwerten größer als 1 extrahiert (vgl. Tabelle 4), die 58,4 \% der Gesamtvarianz erklären. Zur Ergebnisdarstellung wurde die Faktoren-Lösung per Varimax-Rotation mit Kaiser-Normalisierung durchgeführt. Von den insgesamt 19 herangezogenen Variablen wurden 17 mit Faktorladungen $\geq 0,5$ extrahiert, wobei die Variable „Zufriedenheit ehemaliger Patienten“ keinem Faktor zugeordnet werden konnte. Auch die Mitarbeiterzufriedenheit weist in der Faktorladung einen Wert < 0,5 auf. Bei der zusätzlich durchgeführten Reliabilitätsprüfung anhand des Wertes Cronbachs Alpha $(\alpha)$ konnte jedoch ermittelt werden, dass $\alpha$ bei Nichtberücksichtigung der Mitarbeiterzufriedenheit geringer ausfallen würde. Daher wurde diese Variable in die Analyse mit einbezogen.

37 Zur explorativen Faktorenanalyse vgl. einführend z.B. Backhaus u.a. (2003). 


\begin{tabular}{|c|c|c|c|c|}
\hline \multirow{2}{*}{ Variablen } & \multicolumn{4}{|c|}{ Faktoren } \\
\hline & 1 & 2 & 3 & 4 \\
\hline $\begin{array}{l}\text { Qualität der Pflege } \\
\text { nachstationäre Betreuung } \\
\text { persönlicher Zuwendung } \\
\end{array}$ & $\begin{array}{cc}0,796 & \alpha= \\
0,773 & 0,747 \\
0,615 & \\
\end{array}$ & & & \\
\hline $\begin{array}{l}\text { Lage der Klinik } \\
\text { nicht medizinische Ausstattung } \\
\text { Verkehrsanbindung der Klinik } \\
\text { sonstige Ausstattung } \\
\text { Krankenhausbeschreibung allgemein }\end{array}$ & & $\begin{array}{ll}0,779 & \\
0,725 & \\
0,687 & \alpha= \\
0,684 & 0,756 \\
0,537 & \\
\end{array}$ & & \\
\hline $\begin{array}{l}\text { Mortalitätsrate } \\
\text { Krankenhaus-Ranking } \\
\text { Stationäre Wiedereinweisung } \\
\text { Krankenhausinterne Infektionsrate }\end{array}$ & & & $\begin{array}{cc}0,738 & \\
0,732 & \alpha= \\
0,644 & 0,690 \\
0,603 & \\
\end{array}$ & \\
\hline $\begin{array}{l}\text { Qualifikation der Ärzte } \\
\text { Neue Behandlungsmethoden } \\
\text { Mitarbeiterzufriedenheit }\end{array}$ & & & & $\begin{array}{cc}0,843 & \alpha= \\
0,659 & 0,569 \\
0,487 & \\
\end{array}$ \\
\hline
\end{tabular}

Tab. 4: $\quad$ Ergebnis der explorativen Faktorenanalyse und Cronbachs Alpha $(\alpha)$ der zu Faktoren zusammengefassten Items

Quelle: Eigene Darstellung

Die Struktur der für die potenziellen Patienten relevanten Qualitätsinformationen ist gemäß der empirisch ermittelten Faktorlösung eine andere als die Strukturierung der Qualität der Krankenhausleistungen nach den Potenzial-, Prozess- und Ergebnisdimensionen. ${ }^{38}$ Der erste Faktor fasst Variablen von Informationen über die pflegerische Betreuung zusammen, während der zweite Faktor Informationen über nichtmedizinische und nichtpflegerische Aspekte zusammenfasst. Der dritte Faktor umfasst Variablen der objektiven Ergebnisqualität, während der vierte und letzte Faktor Aspekte der medizinischen Behandlung umfasst. Die zuvor in $H 3$ angestellte Überlegung, nach der die Qualitätsinformationen der Qualitätsstruktur der zu Grunde liegenden Leistungen hinsichtlich der Potenzial-, Prozess- und Ergebnisdimension entsprechen, muss somit zurückgewiesen werden.

Auf Basis der zu den Faktoren zusammengefassten Variablen wurde für jeden Faktor ein Indexwert berechnet, ${ }^{39}$ indem die Werte der Variablen eines Faktors für jeden Fall summiert und durch die Anzahl der Variablen geteilt wurden (vgl. Tabelle 5). Für alle Patienten aggregiert ergibt sich, dass die Qualitätsinformationen über die medizinischen Be-

38 Vgl. z.B. Helmig/Dietrich (2001).

39 Alternativ dazu wurde diese Analyse mit den jeweiligen Faktorwerten durchgeführt, was inhaltlich zu vergleichbaren Ergebnissen führte. Da Faktorwerte in gängigen Statistikanwendungen (hier: SPSS) jedoch normiert werden, verlieren sie dadurch einen Teil ihrer Information, da alle Mittelwerte auf 0 und die Standardabweichungen auf 1 gesetzt werden. Zur Bewertung der Wichtigkeit der jeweiligen Faktoren wurde daher mit den ursprünglichen Werten der Variablen gerechnet, die jeweils zu einem Index zusammengefasst wurden. 
handlungsaspekte (Mittelwert 4,28) und Informationen über die pflegerische Betreuung (Mittelwert 4,26) sehr hoch gewichtet werden, gefolgt von objektiven Qualitätsindikatoren (Mittelwert 4,19) und den nichtmedizinischen und nichtpflegerischen Informationen (Mittelwert 3,34). Die nichtmedizinischen und nichtpflegerischen Informationen werden offensichtlich mit Abstand am wenigsten wichtig bewertet, wobei diese aber absolut gesehen nicht unwichtig sind.

\begin{tabular}{|c|c|c|c|c|c|c|}
\hline Faktoren der Information & $N$ & $\begin{array}{l}\text { Mittel- } \\
\text { werte }\end{array}$ & Fälle & $N$ & $\begin{array}{l}\text { Mittel- } \\
\text { werte }\end{array}$ & $\begin{array}{c}\text { Signifikanz } \\
(\text { T-Test })\end{array}$ \\
\hline \multirow{2}{*}{ Faktor 1: pflegerische Betreuung } & \multirow{2}{*}{240} & \multirow{2}{*}{4,26} & leicht & 120 & 4,17 & \multirow{2}{*}{0,059} \\
\hline & & & schwer & 120 & 4,35 & \\
\hline \multirow{2}{*}{$\begin{array}{l}\text { Faktor 2: nichtmedizinische und nicht- } \\
\text { pflegerische Qualitätsaspekte }\end{array}$} & \multirow{2}{*}{240} & \multirow{2}{*}{3,34} & leicht & 120 & 3,51 & \multirow{2}{*}{0,001} \\
\hline & & & schwer & 120 & 3,17 & \\
\hline \multirow{2}{*}{ Faktor 3: Objektive Ergebnisqualität } & \multirow{2}{*}{240} & \multirow{2}{*}{4,19} & leicht & 120 & 4,02 & \multirow{2}{*}{0,000} \\
\hline & & & schwer & 120 & 4,36 & \\
\hline \multirow{2}{*}{$\begin{array}{l}\text { Faktor 4: medizinische Behandlungs- } \\
\text { aspekte }\end{array}$} & \multirow{2}{*}{240} & \multirow{2}{*}{4,28} & leicht & 120 & 4,18 & \multirow{2}{*}{0,018} \\
\hline & & & schwer & 120 & 4,39 & \\
\hline
\end{tabular}

Tab. 5: Angegebene Wichtigkeit von Qualitätsinformationen aus Sicht der Patienten insgesamt und aufgeteilt nach leichten und schweren Fällen

Quelle: Eigene Darstellung

Ein Vergleich der Bewertungen der Wichtigkeit von Qualitätsinformationen zwischen den beiden Gruppen der leichten und schweren Fälle mittels t-Test ergibt, dass die Bewertungen des ersten Faktors auf dem 0,1-Niveau, die Bewertungen des Faktors 4 auf dem 0,05-Niveau und die der Faktoren 2 und 3 auf dem 0,001-Niveau signifikant unterschiedlich sind. Die Hypothese $H 4$, gemäß der die Bedeutung der Qualitätsinformationen je nach Fallschwere unterschiedlich gewichtet werden, kann somit als bestätigt angesehen werden. Für schwere Fälle stellen der Faktor der pflegerischen Betreuung, der medizinischen Behandlung und der objektiven Outcome-Kriterien gleichermaßen wichtige Qualitätsinformationen dar, während nichtpflegerische und nichtmedizinische Qualitätsinformationen deutlich weniger wichtig sind. Im Vergleich hierzu werden von der Gruppe der leichten Fälle die Qualitätsinformationen zur pflegerischen Betreuung und der medizinischen Behandlungen weniger wichtig erachtet wie auch die objektiven medizinischen Qualitätsinformationen für weniger wichtig erachtet werden. Deutlich wichtiger als den schweren Fällen sind den leichten Fällen Qualitätsinformationen über die nichtpflegerischen und nichtmedizinischen Leitungsmerkmale von Krankenhäusern, wobei jedoch auch diese Informationen von den leichten Fällen insgesamt als am wenigsten bedeutsam angegeben wurden. 
bb) Anforderungen an die Informationskanäle von Qualitätsinformationen aus Patientensicht

Aufgrund der rechtlich restriktiv gehaltenen Kommunikationspolitik stellt sich für Krankenhäuser die Frage, welche Informationskanäle am wirkungsvollsten zur Verbreitung der Qualitätsinformationen genutzt werden können. Nachfolgende Tabelle 6 gibt die Antworten auf die Frage wieder, von wem die Befragten Informationen über die Qualität von Krankenhäusern erhalten möchten. Hierbei wird deutlich, dass die (Haus- bzw. Fach-) Ärzte von insgesamt 91,8 \% aller Befragten als Informationsquelle herangezogen würden.

\begin{tabular}{|c|c|c|c|c|c|c|}
\hline \multirow{2}{*}{$\begin{array}{l}\text { Beabsichtigte aktive } \\
\text { Nutzung von Informa- } \\
\text { tionskanälen (Mehr- } \\
\text { fachnennungen mög- } \\
\text { lich) }\end{array}$} & \multicolumn{2}{|c|}{$\begin{array}{l}\text { über alle } \\
(n=462)\end{array}$} & \multicolumn{2}{|c|}{$\begin{aligned} & \text { Fall }=\text { leicht } \\
&(n=178)\end{aligned}$} & \multicolumn{2}{|c|}{$\begin{array}{c}\text { Fall }=\text { schwer } \\
\quad(n=284)\end{array}$} \\
\hline & $\begin{array}{c}\text { Prozent } \\
\text { der Nen- } \\
\text { nungen }\end{array}$ & $\begin{array}{l}\text { Prozent } \\
\text { der Fälle }\end{array}$ & $\begin{array}{c}\text { Prozent } \\
\text { der Nen- } \\
\text { nungen }\end{array}$ & $\begin{array}{c}\text { Prozent } \\
\text { der Fälle }\end{array}$ & $\begin{array}{c}\text { Prozent } \\
\text { der Nen- } \\
\text { nungen }\end{array}$ & $\begin{array}{l}\text { Prozent } \\
\text { der Fälle }\end{array}$ \\
\hline Presse & $3,9 \%$ & $9,2 \%$ & $2,8 \%$ & $5,7 \%$ & $4,6 \%$ & $12,0 \%$ \\
\hline (Haus-, Fach-) Arzt & $38,7 \%$ & $91,8 \%$ & $43,8 \%$ & $89,7 \%$ & $35,6 \%$ & $93,5 \%$ \\
\hline Internet & $14,7 \%$ & $34,9 \%$ & $11,8 \%$ & $24,1 \%$ & $16,5 \%$ & $43,5 \%$ \\
\hline sonstiges & $2,2 \%$ & $5,1 \%$ & $2,2 \%$ & $4,6 \%$ & $2,1 \%$ & $5,6 \%$ \\
\hline $\begin{array}{l}\text { Freunde, Verwandte, } \\
\text { Bekannte }\end{array}$ & $25,1 \%$ & $59,5 \%$ & $25,3 \%$ & $51,7 \%$ & $25,0 \%$ & $65,7 \%$ \\
\hline $\begin{array}{l}\text { Anforderung von In- } \\
\text { formationen }\end{array}$ & $8,4 \%$ & $20,0 \%$ & $8,4 \%$ & $17,2 \%$ & $8,5 \%$ & $22,2 \%$ \\
\hline \multirow[t]{2}{*}{ Tag der offenen Tür } & $6,9 \%$ & $16,4 \%$ & $5,6 \%$ & $11,5 \%$ & $7,7 \%$ & $20,4 \%$ \\
\hline & $100 \%$ & $236,9 \%$ & $100 \%$ & $204,6 \%$ & $100 \%$ & $263,0 \%$ \\
\hline
\end{tabular}

Tab. 6: Antworten auf die Frage nach der beabsichtigten aktiven Nutzung von Informationskanälen bei Notwendigkeit eines Krankenhausaufenthalts

Quelle: Eigene Darstellung

Auch wenn weiter oben ermittelt werden konnte, dass sich die potenziellen Patienten zum größten Teil selbst die Wahl eines entsprechenden Krankenhauses vorbehalten wollten (44,6 \% aller Fälle), so wird doch die Bedeutung der Ärzte als Informationsquellen über Krankenhäuser und deren Qualität mit 91,8 \% aller Fälle klar unterstrichen. Als zweithäufigstes wurden mit 59,5 \% Freunde, Bekannte und Verwandte genannt, während an dritter Stelle mit 34,9\% das Internet als die relevante Informationsquelle genannt wurde. Mit $20 \%$ der Nennungen spielt die Anforderung von Informationsmaterial von Krankenhäusern wie auch mit 16,4\% der Tag der offenen Tür von Krankenhäusern noch eine gewisse Rolle. Informationen über die Presse und sonstige Informationsquellen wie z.B. Radio oder Fernsehen sind hingegen kaum relevant. Eine Analyse des Antwortverhaltens 
aufgeteilt nach der Fallschwere ergibt keinen signifikanten Unterschied zwischen der Gruppe der leichten und der Gruppe der schweren Fälle (Signifikanz =0,517).

Um die Möglichkeiten zu untersuchen, anhand derer Qualitätsinformationen initiativ seitens der Krankenhäuser ohne Aktivitäten von potenziellen Patienten verbreitet werden könnten, wurde weiter analysiert, wie bzw. von wem die potenziellen Patienten Informationen zu Krankenhäuser erhalten möchten, ohne diese explizit anzufordern (vgl. Tabelle 7). Auch hier wurden Haus- bzw. Fachärzte von 35,4 \% der Befragten als die wichtigsten Kanäle angegeben. Wichtig und abweichend von den bisherigen Ergebnissen ist jedoch, dass mit 22,8 \% die Krankenkassen als zweithäufigste Antwort auf diese Frage angegeben wurden. Mit einem vergleichbaren Abstand wurde das Internet als Informationsquelle $(13,3 \%)$ wie auch die Presse $(12,0 \%)$ angegeben, während unabhängige Institute mit 8,9\% und die Krankenhäuser selber mit 7,6\% der Nennungen relativ gesehen für weniger wichtig erachtet werden. Der Vergleich der Häufigkeitsverteilungen zwischen der Gruppe der leichten und schweren Fälle anhand des Chi-Quadrat-Unabhängigkeitstests ergab mit einem Signifikanzwert von 0,614 keinen signifikanten Unterschied zwischen diesen Gruppen. Die Gültigkeit von Hypothese H4, die einen solchen Unterschied unterstellte, muss anhand der Daten somit relativiert werden.

\begin{tabular}{|l|c|c|}
\hline \multicolumn{1}{|c|}{ Informationskanal } & Absolute Häufigkeiten & $\begin{array}{c}\text { Relative Häufigkeiten } \\
\text { (Prozent der Nennungen) }\end{array}$ \\
\hline (Haus-, Fach-) Arzt & 56 & $35,4 \%$ \\
\hline Krankenkasse & 36 & $22,8 \%$ \\
\hline Internet & 21 & $13,3 \%$ \\
\hline Presse & 19 & $12,0 \%$ \\
\hline Unabhängige Institute/Einrichtungen & 14 & $8,9 \%$ \\
\hline Krankenhäuser & 12 & $7,6 \%$ \\
\hline \multirow{2yy}{*}{} & \multicolumn{2}{|c|}{ Signifikanz Chi-Quadrat-Unabhängigkeitstest: 0,614} \\
\cline { 2 - 3 }
\end{tabular}

Tab. 7: Antworten auf die Frage nach Informationskanälen, über die potenzielle Patienten ohne explizite Anforderung informiert werden möchten

Quelle: Eigene Darstellung 


\section{Zusammenfassung und Ausblick}

Mit der vorliegenden Analyse wird die Wettbewerbsrelevanz der Informationspolitik von Krankenhäusern bezüglich der Qualität ihrer Leistungen deutlich. Wie dargestellt, besitzt die Fähigkeit eines Krankenhauses, Qualitätsinformationen wirkungsvoll zu vermitteln, eine in zweifacher Hinsicht wichtige Rolle. Erstens kann damit der Informationsstand der Patienten erhöht werden, was einen, wenn auch nicht besonders stark ausgeprägten, so doch signifikant positiven Einfluss auf die wahrgenommene Leistungsfähigkeit von Krankenhäusern aus Sicht der Patienten hat. Diese höhere Zufriedenheit hat eine geringere Wechselabsicht zur Folge, was ein verringertes Informationssuchverhalten der Patienten nach sich zieht. Zweitens kann davon ausgegangen werden, dass Patienten, die keine zufrieden stellenden Erfahrungen mit Krankenhäusern gemacht haben, in Zukunft ein anderes Krankenhaus zu wählen beabsichtigen und somit ein erhöhtes Informationssuchverhalten aufweisen. Hier kann ein auf Grund der Leistungsstrukturen in Frage kommendes Krankenhaus solche Patienten mit Hilfe von bedarfsgerechten Qualitätsinformationen an sich ziehen. Die bedarfsgerechte Ausgestaltung von Qualitätsinformationen weist somit einen patientenbindenden sowie einen patientenakquirierenden Effekt auf.

Wie die Ergebnisse der vorliegenden Studie weiterhin deutlich machen, entspricht das Informationsbedürfnis von Patienten hinsichtlich der Leistungen von Krankenhäusern nicht der Leistungsstrukturierung gemäß der Potenzial-, Prozess- und Ergebnisdimension. Die Struktur der patientenseitigen Informationsbedürfnisse lässt sich anhand der Dimensionen pflegerische Betreuung, strukturelle nichtmedizinische und nichtpflegerische Leistungsaspekte, objektive Ergebnisqualität sowie medizinische Behandlungsaspekte bilden. Als wesentlichste Qualitätsinformationen werden hierbei die medizinischen Behandlungsaspekte sowie die pflegerische Betreuung angesehen, gefolgt von objektiven Ergebnisindikatoren. Als relativ gesehen weniger bedeutend werden die strukturellen nichtmedizinischen und nichtpflegerischen Leistungsaspekte von Krankenhäusern erachtet. Die Rangreihenfolge der Gewichtung Qualitätsinformationen unterscheidet sich hierbei nicht zwischen der Gruppe der leichten und der schweren Fälle, auch wenn die jeweiligen Bedeutungsangaben in ihrer Höhe einen signifikanten Unterschied aufweisen. Daher erscheint es im Licht der Ergebnisse der vorliegenden Studie nicht notwendig, dass die Gruppe der leichten und der schweren Fälle als unterschiedliche Segmente der Zielgruppe Patienten aufzufassen sind. Bezüglich der bevorzugten Informationskanäle besteht zwischen den leichten und schweren Fällen kein signifikanter Unterschied. Eine gemeinsame Bedienung von leichten und schweren Fällen über dieselben Informationskanäle erscheint daher als angemessen, was ein wichtiges Ergebnis hinsichtlich der Ausgestaltung der Informationspolitik von Krankenhäusern ist.

Bezüglich der Informationskanäle wird jedoch deutlich, dass die Krankenhäuser selbst einen nur geringen direkten Einfluss auf die Verbreitung von Qualitätsinformationen haben, da als bevorzugte Informationskanäle insbesondere die (Haus- und Fach-) Ärzte sowie Freunde, Bekannte und Verwandte genannt wurden. Besteht bei niedergelassenen 
Ärzten noch eine gewisse Beeinflussbarkeit des Informationsverhaltens, welches letztendlich jedoch nicht gänzlich kontrolliert werden kann, so besteht bei der Verbreitung von Qualitätsinformationen durch Freunde, Bekannte und Verwandte nahezu keine Einflussmöglichkeit mehr. Das Problem der mangelnden Beeinflussbarkeit gilt auch für nicht explizit aufgeforderte Informationen, bei denen die Befragten ebenfalls vor allem durch (Haus- und Fach-) Ärzte sowie durch Krankenkassen unaufgefordert informiert werden wollen.

Eine Möglichkeit der direkten Gestaltung von Qualitätsinformationen durch Krankenhäuser stellt allenfalls das Internet dar. Bezüglich der bevorzugten Informationskanäle bei aktiver Informationssuche wie auch bei der nicht aufgeforderten Information über die Leistungsqualität von Krankenhäusern wurde dieses Medium an dritthäufigster Stelle genannt. ${ }^{40}$ In seiner Bedeutung für die Informationspolitik spielt das Internet für Krankenhäuser jedoch weniger hinsichtlich der Erwünschtheit durch potenzielle Patienten eine Rolle, sondern vielmehr, weil Qualitätsinformationen durch Krankenhäuser über diesen Kanal selbst gestaltet werden können.

Die Ergebnisse machen somit deutlich, dass bezüglich der Informationspolitik von Krankenhäusern den Informationsintermediären (Haus- und Fach-) Ärzte sowie den Krankenkassen eine hohe Bedeutung beizumessen ist. Da hinsichtlich der Verpflichtung innerhalb des SGB V zur Qualitätsinformation insbesondere die objektiven Qualitätsindikatoren Berücksichtigung in den publizitätspflichtigen Qualitätsberichten finden, stellt die Verbreitung von weiteren, über die Pflichtangaben hinausgehenden Qualitätsinformationen prinzipiell ein Profilierungspotenzial für Krankenhäuser dar. Die Versorgung der Informationsintermediäre mit entsprechenden Informationsinhalten sollte für Krankenhäuser mit dem Bemühen einhergehen, die Intermediäre zur zielkonformen Weitergabe dieser Qualitätsinformationen zu bewegen. Hinsichtlich der Freunde, Bekannten und Verwandten von potenziellen Patienten sind die Krankenhäuser darauf angewiesen, mit einer möglichst guten Leistung und einer hohen Behandlungsqualität eine positive Mundzu-Mund-Kommunikation und damit eine möglichst hohe Weiterempfehlung auszulösen. $^{41}$

Etwas verwundern muss die Beobachtung, dass die direkt von Krankenhäusern selbst kommunizierten Qualitätsinformationen aus Sicht der Befragten gegenwärtig eine nur geringe Bedeutung aufweisen. Obwohl in anderen Studien bereits nachgewiesen werden konnte, dass ein Bedarf an Information durch Werbung von Krankenhäusern auch unter der deutschen Bevölkerung existiert, ${ }^{42}$ mag das hier beobachtete Ergebnis möglicherweise an der mangelnden Erfahrung von potenziellen Patienten mit dieser Form der Informationsvermittlung durch Krankenhäuser liegen. Diese besaßen bisher nur wenige Möglichkeiten, selbst informative Werbung über ihre Leistungsfähigkeit zu betreiben.

40 Bei der nicht aufgeforderten Information durch das Internet kann es sich hierbei nur um Aktivitäten wie z.B. Newsletter oder andere Informationstechnologien handeln, deren Zusendung und Übermittlung jedoch von potenziellen Patienten zugelassen werden muss.

41 Vgl. Helmig/Dietrich (2001), S. 326 f.

42 Tscheulin/Helmig (1996), S. 1372. 
Als weiteres Forschungsfeld erscheint es daher lohnenswert zu untersuchen, wie der Diffusionsprozess von Qualitätsinformationen abläuft und ob bzw. inwieweit dieser Diffusionsprozess von Krankenhäusern gesteuert oder kontrolliert werden kann. Da die Diffusion von Qualitätsinformationen von Krankenhäusern hauptsächlich über Intermediäre erfolgt, stellt sich für Krankenhäuser die Frage, auf welche Art und Weise die Qualitätsinformationen über diese Intermediäre wirkungsvoll an die Zielgruppe der potenziellen Patienten weitergeleitet werden können. Hierbei ist von besonderem Interesse, wie die Intermediäre dazu gebracht werde können, Qualitätsinformationen im Sinne der Krankenhäuser zu vermitteln und welches Kommunikationsverhalten die Intermediäre aufweisen. Damit verbunden ist die Frage, ob das hier untersuchte beabsichtigte Wechselverhalten mit dem tatsächlichen Wechselverhalten von Patienten übereinstimmt und ob dieses mit dem Diffusionsprozess von Qualitätsinformationen in Verbindung gebracht werden kann. Diese Fragen stellen ein interessantes Gebiet für weitere Untersuchungen auf dem Gebiet der Qualitätsinformationen von Krankenhäusern dar, die sich darüber hinaus auch auf weitere Anbieter medizinischer Leistungen ausweiten lassen.

\begin{abstract}
Martin Dietrich and Oliver Gapp, Quality Information of Hospitals - an Analysis of Relevance and Patients' Requirements
\end{abstract}

Competition; health care economics; hospital management; information policy in health care; Quality information of hospitals; patients' choice behaviour

With regard to the increasing pressure of competition among hospitals quality belongs to the strongest arguments to influence patients' choice behavior to ensure hospitals' occupancy rates. Besides securing a high quality hospitals are challenged to communicate their quality effectively. As especially patients as the ultimate users of the health care systems form the very target group of hospitals it is of major interest of what relevance quality information are to hospitals due to the patients intended choice behavior, what information about hospitals' quality patients request and what information channels of quality patients are likely to use. Furthermore it is of interest if the severity of illness influences the requested quality information and the preferred channels of quality information. Results of a study among 240 potential patients are presented. 


\section{Literaturverzeichnis}

Adam, Dietrich und Petra Gorschlüter (1999), Qualitätsmanagement im Krankenhaus, in: Zeitschrift für Betriebswirtschaft, Ergänzungsheft 5/99, S. 95-109

Backhaus, Klaus u.a. (2003), Multivariate Analysemethoden, Eine anwendungsorientierte Einführung, 10. Aufl., Berlin

Bem, Daryl J. (1972), Self-perception theory, in: Advances in Experimantal Social Psychology, Vol. 6, S. 2-62

Beumers, Angela und Peter Borges (1997), Was erwarten niedergelassene Ärzte von Krankenhäusern?, in: führen und wirtschaften im Krankenhaus, 14. Jg., H. 3, S. 221-223

Borges, Peter (2003), Kommunikation, in: führen und wirtschaften im Krankenhaus, 20. Jg., Heft 3, S. $267-269$

Boulding, William u.a. (1993), A Dynamic Process Model of Service Quality: From Expectations to Behavoiral Intentions, in: Journal of Marketing Research, Vol. 30, S. 7-27

Chassin, Mark R., Edward L. Hannan und Barbara A. DeBuono (1996), Benefits and hazards of reporting medical outcomes publicly, in: New England Journal of Medicine (NEJM), Vol. 334, No. 6, February, S. 394-398

Cheng, Shou-Hsia, Yi-Chen Ho und Kuo-Piao Chung (2002), Hospital quality information for patients in Taiwan, Can they understand it?, in: International Journal for Quality in Health Care, Vol. 14, No. 2, S. 155-160

Davies, Huw T. O und Martin N. Marshall (1999), Public disclosure of performance data - Does the public get what the public wants?, in: The Lancet, Vol. 353, S. 1639-1640

Donabedian, Avedis (1966), Evaluating The Quality of Medical Care, in: Milbank Memorial Fund Quarterly, Vol. 44, No. 3, S. 166-206

Donabedian, Avedis (1980), The definition of quality and approaches to ist assessment, Vol. 1, Michigan

Ende, Jack u.a. (1989), Measuring Patients' Desire for Autonomy, in: Journal of General Internal Medicine, Vol. 4, S. 23-30

Etienne, Michèle (2000), Total Quality Management im Spital erfolgreich gestalten, Bern

Festinger, Leon (1957), A Theory of Cognitive Dissonance, Stanford (California)

Galvin, Robert S. (1998), Are Performance Measures Relevant?, in: Health Affairs, S. 29-31

Gesetz zur Reform der gesetzlichen Krankenversicherung ab dem Jahr 2000 (GKV-Gesundheitsreform 2000) vom 22. Dezember1999 (BGB1 I, S. 2626)

Halm, Ethan A. und Mark R. Chassin (2001), Why Do Hospital Death Rates Vary?, in: New England Journal of medicine, Vol. 345, No. 9, S. 692-694

Haubrock, Manfred, Norbert Meiners und Frank Albers (1998), Krankenhausmarketing - Analyse, Konzepte, Methoden, Stuttgart

Helmig, Bernd (2001), Ökonomischer Erfolg in öffentlichen Krankenhäusern, bisher unveröffentlichte Habilitationsschrift, Universität Freiburg

Helmig, Bernd und Martin Dietrich (2001), Qualität von Krankenhausleistungen und Kundenbeziehungen - Das Beispiel „Ambulante Krankenhausbehandlungen von Kindern“, in: Die Betriebswirtschaf, 61. Jg., Heft 3, S. 319-334

Herkner, Werner (1993), Lehrbuch der Sozialpsychologie, 5. Aufl., Bern

Herkner, Werner (Hrsg.) (1980), Attribution - Psychologie der Kausalität, Bern

Hibbard, Judith H., Shoshanna Sofaer und Jaquelyn J. Jewett (1996), Condition-Specific Performance Information: Assessing Salience, Comprehension, And Approaches for Communicating Quality, in: Health Care Financing Review, Vol. 18, Iss. 1, S. 95-110

Hibbard, Judith H., Paul Slovic und Jaquelyn J. Jewett, (1997), Informing Consumer Decisions in Health Care: Implications from Decision-Making Research, in: The Milbank Quarterly, Vol. 75, No. 3, S. 395-414

Hribek, Günther und Helmut Schmalen (2000), Konzeptionalisierung und Operationalisierung der Patientenzufriedenheit mit stationärer Versorgung - Entwicklung multiattributiver Messinstrumente für Krankenhäuser und Rehabilitationseinrichtungen, in: Marketing Zeitschrift für Theorie und Praxis, 22. Jg., Heft 3, S. 208-226

Hüttner, Manfred (1997), Grundzüge der Marktforschung, 5. Aufl., München

Javalgi, Rajshekar G., S. R. Rao und Edward G. Thomas (1991), Choosing a Hospital - Analysis of Consumer Tradeoffs, in: Journal of Health Care Marketing, Vol. 11, No. 1, S. 12-22

Johnson, Grace L. und Arkalgod Ramaprasad (2000), Patient-Physician Relationships in the Inforamation Age, in: Marketing Health Services, Vol. 20, No. 1, S. 21-27

Kaltenbach, Tobias (1991), Qualitätsdefinition im Krankenhaus - Ein Beitrag zum Qualitätsverständnis, in: Zeitschrift für öffentliche und gemeinwirtschaftliche Unternehmen, Bd. 14, Heft 4, S. 442-450

Kaltenbach, Tobias (1992), Qualität im Krankenhaus - Eine Managementaufgabe, in: Betriebswirtschaftliche Forschung und Praxis, 54. Jg., Heft 5, S. 473-480

Kaltenbach, Tobias (1993), Qualitätsmanagement im Krankenhaus - Qualitäts- und Effizienzsteigerung auf der Grundlage des Total-Quality-Management, 2. Aufl., Melsungen

Kamakura, Wagner A. u.a. (2002), Assessing the Service-Profit Chain, in: Marketing Science, Vol. 21, S. 294-317

Kurz, Richard S. und Frederic D. Wolinsky, (1985), Who picks the hospital? Practioner or patient?, in: Hospital and Health Service Administration, No. 30, S. 95-106 
Lane, Paul M. und Jay D. Lindquist (1988), Hospital Choice, A Summary of the Key Empirical and Hypthetical Findings of the 1980s, in: Journal of Health Care Marketing, Vol. 8, No. 4, S. 5-20

Lubalin, James S. und Lauren D. Harris-Kojetin (1999), What Do Consumers Want and Need to Know in Making Health Care Choices, in: Medical Care Research and Review, Vol. 56, Supplement 1, S. 67-102

Luft, Harold S. u.a. (1990), Does Quality Influence Choice of Hospital?, in: The Journal of the American Medical Association (AMA), Vol. 263, No. 21, S. 2899-2906

Lüngen, Markus und Karl W. Lauterbach (2002), Zehn Thesen zum Strategiewandel im Krankenhaus, in: Betriebswirtschaftliche Forschung und Praxis, 54. Jg., Heft 2, S. 156-171

Malhotra, Naresh K. (1983), Stochatsic modelling of consumers preferences for health care institutions, in: Journal of Health Care Marketing, Vol. 3, No. 4, S. 18-26

May, Uwe und Jürgen Wasem (2003), Medizinische Risiken versus ökonomische Chancen der gesundheitlichen Eigenverantwortung, in: Gesundheitsökonomie und Qualitätsmanagement, 8. Jg., S. 31-38

McKee, Martin (1997), Indicators of clinical Performance, in: Journal of the British Medical Association (BMJ), No. 315, S. 142-144

McKee, M. und David Hunter (1995), Mortality league tables: do the inform or mislead?, in: Quality in Health Care, No. 4, S. 5-12

Meurer, Uta (1998), Die Krankenhäuser müssen ihre Leistung selbst darstellen, bevor es andere tun, in: führen und wirtschaften im Krankenhaus, 15. Jg., Heft 4, S. 310-313

Morwitz, Vicky und David Schmittlein (1992), Using Segmentation to Improve Sales Forecasts Based on Purchase Intent - Whicht 'Intenders 'Acutally Buy, in: Journal of Marketing Research, Vol. 29, S. 391-405

Mukamel, Dana B. und Alvin I. Mushlin (2001), The Impact of Quality Report Cards on Choice of Physicians, Hospitals, and HMOs: A Midcourse Evaluation, in: Journal of Quality Improvement, Vol. 27, No. 1, S. 20-27

Mukamel, Dana B. und Alvin I. Mushlin (1998), Quality of Care Makes a Difference, in: Medical Care, Vol. 36, No. 7, S. 945-954

Nease, Robert F. und Blair Brooks (1995), Patient Desire for Information and Decision Making in Health Care Decisions - The Autonomy Preference Index and the Health Opinion Survey, in: Journal of General Internal Medicine, Vol. 10, No. 11, S. 593-600

Neubauer, Günter (2004), Zur ökonomischen Steuerung der Krankenhausversorgung unter DRG-Fallpauschalen, in: Krankenhausreport 2003, Schwerpunkt: G-DRGs im Jahre 1, hrsg. von J. Klauber, B.-P. Robra und H. Schellschmidt, S. 101-119

Nix, Thomas W. und Jeffrey G. Gibson (1987), Does a hospital`s religious affiliation affect choice of hospital and patient satisfaction?, in: Journal of Health Care Marketing, Vol. 9, No. 2, S. 40-41

Nutley, Sandra und Peter Smith (1998), League tables for performance improvement in health care, in: Journal of Health Services Research and Policy, Vol. 3, No. 1, S. 50-57

Oberender, Paul und Frank Daumann (1996), Administrierte Qualitätssicherung oder wettbewerbliche Lösung?, in: WiSt, Heft 11, S. 566-571

Olandt, Henrik (1998), Dienstleistungsqualität in Krankenhäusern. Operationalisierung und Messung der Patientenwahrnehmung, Wiesbaden

Olandt, Henrik und Martin Benkenstein (1999), Modelle der Dienstleistungsqualität in Kliniken - Operationalisierung und Validierung auf der Basis von SERVQUAL und TEILQUAL, in: Zeitschrift für BetriebswirtschaftErgänzungsheft 5/99, S. 111-124

Robinson, Sandra und Mollyann Brodie (1997), Understanding the Quality Challenge for Health Consumers - The Kaiser/AHCPR Survey, in: Journal on Quality Improvement, Vol. 23, No. 5, S. 239-244

Sachverständigenrat für die Konzertierte Aktion im Gesundheitswesen (SKAiG) (2001), Gutachten 2000/2001 des Sachverständigenrats für die Konzertierte Aktion im Gesundheitswesen - Bedarfsgerechtigkeit und Wirtschaftlichkeit, Band I: Zielbildung, Prävention, Nutzerorientierung und Partizipation, Berlin

Sachverständigenrat für die Konzertierte Aktion im Gesundheitswesen (SKAiG) (2003), Gutachten 2003 des Sachverständigenrats für die Konzertierte Aktion im Gesundheitswesen - Finanzierung, Nutzerorientierung und Qualität, Band I: Finanzierung und Nutzerorientierung, Berlin

Saeed, Khalid S. B. (1998), Factors affecting patients choice of hospitals, in: Annals of Saudi Medicine, Vol. 18, No. 5, S. 420-424

Schmutte, Andre M. (1998), Total Quality Managemet im Krankenhaus, Wiesbaden

Schrappe, Matthias (1999), Leistungs- und Qualitätsvergleich im Krankenhaus - Zukunft des Krankenhausbetriebsvergleichs?, in: Betriebswirtschaftliche Forschung und Praxis, 51. Jg., Heft 5, S. 499-511

Sloane, Graeme, Paula Tidwell und Melanie Horsfield (1999), Identification of the Decision Maker for a Patient's Hospital Choice - Who Decides Which Hospital?, in: Jouranl of Hospital Marketing, Vol. 13, No. 1, S. 57-77

Smith, Peter (1995), On the unintended consequences of publishing performance data in the public sector, in: International Journal of Public Administration, Vol. 18, No. 2 und 3, S. 277-310

Sozialgesetzbuch - Fünftes Buch (V) - Gesetzliche Krankenversicherung vom 20. Dezember 1988 (SGB V) (BGB1 I, 2477), zuletzt geändert durch Artikel 1 und Artikel 2 des Gesetzes zur Modernisierung der gesetzlichen Krankenversicherung (GKV-Modernisierungsgesetz GMG) vom 19. November 2003 (BGB1 I, S. 2190)

Taylor, Susan L. und Louis M. Capella (1996), Hospital outshopping - Determinant attributes and hospital choice, in: Health Care Management Review, Vol. 21, No. 4, S. 33-44 
Tscheulin, Dieter K. und Bernd Helmig (1996), Arzt- und Krankenhauswerbung. Rechtliche Grundlagen, ,State-ofthe-Art“ und Direktiven für eine effiziente Ausgestaltung, in: Zeitschrift für Betriebswirtschaft, 66. Jg., Heft 11, S. 1357-1382

Tscheulin, Dieter K. und Bernd Helmig (2000), Krankenhausmarketing - Dienstleistungsmarketing, in: Krankenhausmanagement, hrsg. von Peter Eichhorn, Hans-Jürgen Seelos, J.-Matthias Graf von der Schulenburg, München

Tscheulin, Dieter K. und Bernd Helmig (2001), Krankenhausmarketing, in: Branchenspezifisches Marketing, Grundlagen - Besonderheiten - Gemeinsamkeiten, hrsg. von denselben, Wiesbaden, S. 401-428

Tscheulin, Dieter K., Bernd Helmig und Ralf Moog (2001), Die Ermittlung entscheidungsrelevanter Variablen der Wahl von Geburtskliniken als Basis eines effizienten Krankenhausmarketing, in: Zeitschrift für öffentliche und gemeinwirtschaftliche Unternehmen, Bd. 24, Heft 4, S. 451-468

Tuschen, Karl H. und Ulrich Trefz (2004), Krankenhausentgeltgesetz - Kommentar mit einer umfassenden Einführung in die Vergütung stationärer Krankenhausleistungen, Stuttgart

Viethen, Gregor (1995), Qualität im Krankenhaus - Grundbegriffe und Modelle des Qualitätsmanagements, Stuttgart

Wallace, Cathrine (1985), Woman's health-care spending new target of hospitals ads, in: Modern Healthcare, No. 15 , S. $52-56$

Woodside, Arch G., Lisa Frey und Robert T. Daly (1989), Linking Service Quality, Customer Satisfaction and Behavioral Intention, in: Journal of Health Care Marketing, Vol. 9, No. 4, S. 5-17

Woodside, Arch G., Chris M. Sertich und James M. Chakalas (1987), Hospital Choice - patient attribution of the decision and satisfaction with the services, in: Journal of Health Care Marketing, Vol. 7, No. 1, S. 61-68

Zeithaml, Valarie A., Leonard L. Berry und A. Parasuraman (1996), The Behavoiral consequences of Service Quality, in: Journal of Marketing, Vol. 60, S. 31-46 